\title{
The Non Mineral Axis Klotho-Vitamin D in Diabetic Nephropathy: Review
}

Farid Nakhoul ${ }^{1-4 *}$, Nakhoul Nakhoul ${ }^{3}$, Nadia Thaucho ${ }^{3}$, Evgeny Farber ${ }^{2}$, Hao Zhang ${ }^{5}$, Mohamed Awawde ${ }^{6}$, Adi Francis $^{6}$ and Inbal Dahan $^{3}$

${ }^{1}$ Medicine and Biomedical Sciences, The University of Chicago, Chicago, USA

${ }^{2}$ Nephrology and Hypertension Division, The Baruch Padeh Poriya Medical Center, Lower galilee, Israel

${ }^{3}$ Diabetic Nephropathy Laboratory, The Baruch Padeh Poriya Medical Center, Israel

${ }^{4}$ Faculty of medicine in the Galilee, Israel

${ }^{5}$ Central South University, Changsha, China

${ }^{6}$ Nephrology and cardiology divisions, The Holy Family Hospital, Nazereth, Israel

\begin{abstract}
Diabetes Mellitus (DM) is metabolic disease associated with hyperlipidemia and arterial hypertension that are known as major risk factors for coronary heart disease. Diabetic complications are related to increased oxidative stress due to elevated glucose levels in the plasma. DM patients suffer from higher incidence of micro vascular complications such as Diabetic Nephropathy (DN). DN is the leading cause of End Stage Renal Disease (ESRD) and accounts for approximately $40 \%$ of all patients who require replacement therapy. Recently, the proximal tubular damage caused by increased deposition of iron in the lysosomes accompanied with oxidative stress, was described as another mechanism involved in the pathogenesis of DN. The well-known risk factors for DN are uncontrolled glucose levels, genetic factors such as Haptoglobin (Hp) and Angiotensin Converting Enzyme (ACE) genes polymorphism. Despite the availability of various drugs for DN such as converting enzyme inhibitors (ACEIs), angiotensin receptor blockers (ARB) and renin inhibitors (RI), 40\% of type 2 DM (T2D) patients will develop ESRD. Hence, the aim of this review is to understand the importance of klotho protein and vitamin $D$ in the pathogenesis of DN and as a potential future target for treatment.
\end{abstract}

Keywords: Cardio-vascular complication; Diabetic nephropathy; Klotho; Vitamin D; Vitamin D receptor

Abbreviations: ACE: Angiotensin Converting Enzyme; ACEIs: Angiotensin Converting Enzyme Inhibitors; AgI: Angiotensin I; Ag-II: Angiotensin II; CKD: Chronic Kidney Disease; DM: Diabetes Mellitus; T1D: type 1 DM; T2D: type 2 DM; DN: Diabetic Nephropathy; eGFR: Estimated Glomerular Filtration Rate; ESRD: End Stage Renal Disease; FGF-23: Fibroblast Growth Factor-23; FGFR1: Fibroblastic Growth Factor-receptor; HG: High Glucose; Hp: Haptoglobin; Hvdr: Human VDR; Mtor: Mammalian target of rapamycin; NF-kB: the nuclear factor $\beta$; RAS: Renin-Angiotensin System; RAAS: Renin-AngiotensinAldosterone System; RI: Renin Inhibitor; ROS: Reactive Oxygen Species; STZ: Streptozotocin; Tg: Transgenic; TGF $\beta$ : Transforming Growth Factor $\beta$; VDR: Vitamin D Receptor; VDRA: Vitamin D Receptor Activator; $1,25(\mathrm{OH})_{2} \mathrm{D}_{3}$ : 1,25-dihydroxyvitamin D3

\section{Introduction}

Diabetic nephropathy (DN) is the most common renal complication of diabetes mellitus (DM) and the leading cause of End Stage Renal Disease (ESRD) and dialysis treatments [1-4]. The tissue renin angiotensin aldosterone system (RAAS) is well establishes as mediator of renal fibrosis especially via the angiotensin II (Ag-II) and aldosterone effect, particularly in DM [5]. In early stages of DM there is enormous increase in glomerular filtration rate via increased production of Ag-II and decreased production of nitric oxide. In vitro studies showed that when exposed to high glucose levels, mesangial cells and podocytes increases renin and Ag-II production [5,6]. High glucose increased free radicals production via Fenton reaction, with membrane damage. Ag-II also induces increased in oxidative stress, synthesis of pro inflammatory factors with increased glomerular and tubular cell damage. The increased production of renin, Ag-II, and proinflammatory factors, can accelerate the fibrogenesis and finally diffuse glomerulosclerosis $[5,7,8]$.

Recently calcitriol, the active compound of vitamin D, the 1,25-dihydroxyvitamin $\mathrm{D} 3\left(1,25(\mathrm{OH})_{2} \mathrm{D}_{3}\right)$, has been shown to function as protective pathway during hyperglycemic state, functioning as negative endocrine regulator of the RAAS by suppressing the renin gene transcription [9-12]. These so-called non-calcemic activities include regulation of renal and cardiovascular functions and modulation of immune response. The molecular basis for the broad functionalities of vitamin $\mathrm{D}$ is the expression of the vitamin $\mathrm{D}$ receptor (VDR) in virtually all tissues in the body. The activities of $1,25(\mathrm{OH})_{2} \mathrm{D}_{3}$ are mediated by the VDR, a member of the nuclear receptor superfamily [10-14]. Relevant examples of these non-calcemic activities are regulation of the RAAS, and the nuclear factor B (NF-kB) pathway, two pathways involved in a broad range of pathological processes such as DN $[12,13,15]$.

The genetic null mutant mice lacking the VDR develop hyperreninemia, hypertension and proteinuria. In type I DM (T1D), VDR knockout mice develop more severe glomerulopathy than wild type [15]. On the other hand, klotho protein, present in the distal and proximal convolute tubules, as well as the secreted klotho regulates multiple activities, including suppression of growth factor signaling, synthesis of $1,25(\mathrm{OH})_{2} \mathrm{D}_{3}$, regulation of several ion channels, and suppression of oxidative stress [16]. As bioactive molecule, protects cells from senescence and apoptosis, especially in DM patients [16].

Thus, this review will promote the current understanding of the molecular biology of klotho, the cross talk with active vitamin $\mathrm{D}$, and

${ }^{*}$ Corresponding author: Farid Nakhoul, Medicine and Biomedical Sciences, The University of Chicago, 900E, 57th Street, KCBD 9125, Chicago, IL 60637 USA, Tel: +972 246653587; E-mail: Fnakhoul1@medicine.bsd.uchicago.edu

Received May 18, 2015; Accepted June 13, 2015; Published June 20, 2015

Citation: Nakhoul F, Nakhoul N, Thaucho N, Farber E, Zhang H, et al. (2015) The Non Mineral Axis Klotho-Vitamin D in Diabetic Nephropathy: Review. J Diabetes Metab 6: 563. doi:10.4172/2155-6156.1000563

Copyright: ( 2015 Nakhoul F, et al. This is an open-access article distributed under the terms of the Creative Commons Attribution License, which permits unrestricted use, distribution, and reproduction in any medium, provided the original author and source are credited. 
will present future directions for klotho-vitamin D research in the field of DN.

\section{Klotho Protein}

Klotho, is a novel anti-aging gene encoding a protein with a multiple pleiotropic effects. The klotho family of proteins consists of three members: $\alpha$ klotho, $\beta$ Klotho and $\gamma$ klotho. All three are single-pass transmembrane proteins. The founder klotho is designated aklotho to distinguish it from the other two members. aKlotho gene is composed of five exons, in humans, mice and rats, is highly expressed in the distal and proximal convolute tubular epithelium of normal adult kidneys [16-18]. Also is expressed in pancreas, parathyroid and brain. The renal klotho gene expression is regulated by pathophysiological conditions including DM especially in DN and cardio-vascular disease $[16,19]$.

aKlotho is encoded as a single-pass transmembrane protein with a molecular weight of $130 \mathrm{kDa}$, and it functions as the co-receptor for fibroblast growth factor-23 (FGF-23) [17,18,20], a bone-derived hormone that plays a critical role in phosphate homeostasis and vitamin D biosynthesis in the kidney $[19,20]$. Klotho is further functions as an anti-aging protein that extends life span when over-expressed, suppresses growth factor signaling and oxidative stress, accelerates aging-like phenotypes when disrupted in mice [21,22]. Klotho protein exists in two forms: A membrane and secreted form [17]. The soluble klotho is further well known as anti-apoptotic activity especially in experimental ischemic acute kidney injury [21].

Transmembrane klotho is a cofactor that converts fibroblastic growth factor-receptor 1 (FGFR1) into a specific receptor for FGF23 and decreases $1,25(\mathrm{OH})_{2} \mathrm{D}_{3}$ synthesis in the kidney. $1,25(\mathrm{OH})_{2} \mathrm{D}_{3}$ stimulates both klotho and FGF-23, and both FGF-23 and klotho inhibits $1,25(\mathrm{OH})_{2} \mathrm{D}_{3}$ via $1 \alpha$-hydroxylase. It has also been reported that the VDR controls expression of the klotho gene. Up regulation or restoration of klotho by $1,25(\mathrm{OH})_{2} \mathrm{D}_{3}$ may provide a means to slow down the progression of chronic kidney disease (CKD) and improve cardiovascular disease especially in the DM patients $[16,17,23]$.

The over expression of klotho in a mouse model of glomerulonephritis restored mitochondrial function and suppressed mitochondrial DNA damage in kidney. It also suppresses apoptosis in the hyperglycemic state, resulting in preservation of renal function [24].

The klotho protein has also endogenous anti fibrotic function via antagonism of $\mathrm{Wnt} / \beta$-catenin signaling, which promotes fibrogenesis, suggesting that loss of Klotho may contribute to the progression of DN by accelerated fibrogenesis [24,25]. In addition to the effect of klotho on Transforming Growth Factor $\beta$ (TGF- $\beta$ ), suppressive effects of klotho on the insulin-like growth factor pathway may be associated with inhibitory action on renal fibrosis [25-28] and cardio-renal protection in high oxidative stress condition such as diabetes and its complications.

\section{Decreased Renal Klotho Expression in Early DN in Humans and Mice}

It is generally accepted that klotho expression in the kidney is markedly decreased in the early stages of DN and in patients with CKD $[23,29]$. Although the exact mechanism of how klotho is reduced in diabetic kidney disease is not well understood, the increased oxidative stress, Ag-II and TGF $\beta-1$ may be involved $[22,26]$. As we reported in our previous work, the hyperglycemic state is accompanied by increased production of reactive oxygen species (ROS) by the Fenton reaction $[3,4]$.
Hyperglycemia is an important factor in the induction of DN. High blood glucose induces excessive production of ROS in the renal mesangial and tubular cells causing membrane damage [22]. Cheng and his group [30] investigated the effect of insulin and floridzin on hyperglycemia in DM rats, especially klotho expression. Both insulin and floridzin reversed the lower klotho expression levels in diabetic kidneys.

Osamu Asai and his group [29] had published a paper on the role of klotho in the DN mice. In this study, they showed that renal Klotho expression levels were decreased in patients with early DN and in the streptozotocin (STZ) induced mouse model of T1D. In patients with minimal change glomerulopathy and IgA nephropathy, the reactivity was significantly reduced in samples from DN patients correlated with estimated glomerular filtration rate (eGFR), suggesting that renal Klotho mRNA expression levels were decreased with the progression of renal failure.

Other study by Lee and his group [31] were the first to demonstrate that plasma and urine levels of soluble Klotho are significantly elevated in the DM patients with relatively preserved renal function compared to control subjects. Hence, in DN, klotho expression levels were decreased in kidneys of patients with early DN but with increased levels of plasma and urinary klotho, and that klotho deficiency may serve as a biomarker as well as a pathogenic factor for the progression of renal disease and further complications [30,31].

In vivo studies had shown that there is a therapeutic potential of klotho in several kidney disease models including DN in mice model $[32,33]$. Klotho administration has been proven successful in the protection of kidney function in acute kidney injury in animals induced by ischemia- reperfusion injury and high glucose induced glomerular damage $[22,25]$

\section{Genetic Deficiency of Klotho, Exacerbates Early Nephropathy in STZ-induced DM in Mice}

The recent characterization of the klotho protein that modulates the expression level of antioxidant enzymes, as well as its high expression level in the kidney, suggest that klotho plays a role in accelerated aging and cellular senescence observed in DM $[16,17]$. More striking klotho-deficient mice exhibit multiple age-related phenotypes such as premature death $[28,29]$ whereas mutation of the klotho gene caused multiple premature-aging phenotypes and shortened life span [34]. Klotho gene mutant ( $\mathrm{KL}+/-$ ) and wild-type mice (6-8 weeks) were injected with multiple low doses of STZ, which known to cause selective $\beta$ cell destruction and subsequently modest hyperglycemia leading to functional damage (albuminuria) and histological lesions in kidneys [35]. The purpose of this study by Yi Lin and his group [35] was to investigate the hypothesis that klotho gene deficiency enhances TGF- $\beta 1$, and Mammalian target of rapamycin (mTOR) signaling and promotes the development of early DN. One of the novel findings of this study was that renal klotho deficiency in Klothodeficient mice (KL-/-) exacerbated early DN (impaired renal function and glomerular meningeal matrix expansion) in T1D induced by STZ [35]. Hyperglycemia by itself is not sufficient to cause this severe renal damage. However klotho deficiency and hyperglycemia can causes oxidative damage that may contribute to the exacerbation of DN.

\section{Vitamin D}

The role of Vitamin D in the treatment of early stages of $\mathrm{DN}$ is not well understood or proven. The inactive vitamin $\mathrm{D}$ is transported to the liver, where it is hydroxylated in the 25 position 
to yield 25-hydroxyvitamin $\mathrm{D}$, which is the major circulating form of vitamin $\mathrm{D}$. The 25 -hydroxyvitamin $\mathrm{D}$ is further hydroxylated by 1 - $\alpha$-hydroxylase in the proximal convolute tubule of the kidney, to yield $1,25(\mathrm{OH})_{2} \mathrm{D}_{3}$, which is the active form of vitamin $\mathrm{D}[36]$. The activities of $1,25(\mathrm{OH})_{2} \mathrm{D}_{3}$ are mediated by the VDR, a member of the nuclear receptor superfamily. The most well-known function of the vitamin $\mathrm{D}$ endocrine system is to maintain calcium and phosphorus homoeostasis; however, studies in the past decades have unveiled a wide range of activities for vitamin $\mathrm{D}$ that extends beyond this function. These so-called non-calcemic activities include regulation of renal and cardiovascular functions and modulation of immune response. Relevant examples of these non-calcemic activities are regulation of the RAAS and the nuclear factor NF- $\kappa B$ pathway, two pathways involved in a broad range of pathological processes [37]. In the kidney, vitamin D is important for maintaining podocytes integrity, suppressing renin gene expression and inflammation. Therefore, vitamin $\mathrm{D}$ has the potential to have a favorable impact in DN via VDR in kidney protection from DM oxidative stress injury [38].

\section{Pathways involving vitamin D: The Renin-Angiotensin- Aldosterone System (RAAS)}

Vitamin D is a negative endocrine regulator of the RAAS [37,39]. Li and his group were the first to demonstrate that $1,25(\mathrm{OH})_{2} \mathrm{D}_{3}$ directly suppressed both plasma renin expression and activity of the renin gene promoter in cell cultures $[37,38]$. They also provided in vivo evidence that inhibition of $1,25(\mathrm{OH})_{2} \mathrm{D}_{3}$ in wild-type mice led to a significant increase in the expression of renin mRNA and increased plasma renin levels. Correspondingly, treatment of wild-type mice with $1,25(\mathrm{OH})_{2} \mathrm{D}_{3}$ suppresses renin transcription [9].

The intra-renal RAS plays a key role in diabetic renal injury. The Ag-II, the effector of the RAS, is known to promote podocyte apoptosis in vitro and in vivo [40]. Glomerular renin was upregulated in DM wild type mice, and the human VDR transgene, blocked the increase in renin expression, particularly in the presence of the active vitamin D compounds. In podocyte cultures, high glucose (HG) induced the expression of renin and angiotensinogen. As a result intracellular renin activity and released extracellular Ag-II levels in the media. These induction were blocked by $1,25(\mathrm{OH})_{2} \mathrm{D}_{3}$. Moreover, HGinduced angiotensin $1(\mathrm{Ag} 1)$ receptor expression in podocytes was also attenuated by $1,25(\mathrm{OH})_{2} \mathrm{D}_{3}$. These data suggest that the $1,25(\mathrm{OH})_{2} \mathrm{D}_{3}$ VDR signaling inhibits the RAAS activation in podocytes, and suppress HG-induced podocyte apoptosis [5,41,42]. Vitamin D is further involved in cell differentiation and anti-proliferative pathways and plays a key role in immunity, vascular function, cardio myocyte health, insulin resistance, and modulation of RAS. Consequently, vitamin $\mathrm{D}$ deficiency has been associated with arterial hypertension, DM and a higher frequency of cardiovascular disease and cardiovascular mortality [41].

\section{Reno protective roles of Vitamin D-VDR signaling}

Numerous observational studies showed multiple beneficial effects of $1,25(\mathrm{OH})_{2} \mathrm{D}_{3}$ or vitamin $\mathrm{D}$ analog therapy in patients with kidney disease especially DN that led to a significant survival advantage for patients receiving the therapy. A key reno protective function of vitamin $\mathrm{D}$ is to reduce albuminuria or proteinuria, a major risk factors for CKD progression, renal failure, cardiovascular events, and death $[5,42,43]$. This anti-proteinuric effect and slowing the progression of $\mathrm{DN}$ is mediated primarily via the RAAS.

$\mathrm{Li}$ and his group [14] has shown that in a DM state or under unilateral ureteral obstruction, genetic deletion of VDR in mice leads to severe renal injury largely owing to increased activation of the local RAS in the kidney, resulting in early onset and robust albuminuria, glomerulosclerosis, and interstitial fibrosis. They also showed that podocytes express the VDR that is highly inducible by $1,25(\mathrm{OH})_{2} \mathrm{D}_{3}$, and through VDR, $1,25(\mathrm{OH})_{2} \mathrm{D}_{3}$ transcriptionally stimulates the expression of nephrin, a key slit diaphragm protein synthesized by podocytes [14].

Wang [44] used the $2.5-\mathrm{kb}$ human podocin gene promoter to target Flag-tagged human VDR (hVDR) to podocytes in DBA/2J mice, a genetic background known to be susceptible to diabetic renal injury. This podocin gene promoter has been well documented for its podocyte specificity in driving transgene expression. To test whether an increase in podocyte VDR would enhance the renoprotective effect of vitamin $\mathrm{D}$, they compared the therapeutic efficacy of a lowdose vitamin D analog doxercalciferol between STZ-induced diabetic wild-type (WT) and hVDR transgenic (Tg) mice. Thus, podocyte VDR overexpression renders the mice more sensitive to vitamin $\mathrm{D}$ therapy. In fact, TG mice showed significantly reduced albuminuria compared with WT mice even at baseline. Thus, they provide mechanistic insights into the ever-increasing epidemiologic and clinical evidence linking vitamin D deficiency to renal and cardiovascular problems, and provide molecular basis to explore therapeutic use of vitamin $\mathrm{D}$ and its analogs in the prevention and intervention of these diseases.

The synergistic therapeutic effects of combined vitamin D analog Paricalcitol (19-0nor-1,25-dihydroxyvitamin D2) with AT1 receptor antagonist (Losartan) on kidney disease in a model of type 2 diabetic mice, showed a dramatic therapeutic synergism, manifested by prevention of progressive albuminuria, restoration of the glomerular filtration barrier, reversal of the decline in slit diaphragm proteins, and reduction of glomerulosclerosis [45].

\section{Vitamin D and Klotho cross-talk}

The kidney is not only excretory organ but also produce several active hormones such as erythropoietin, $1,25(\mathrm{OH})_{2} \mathrm{D}_{3}$, renin and klotho. Klotho exerts multiple actions on the kidney, and the most important is regulation of $1,25(\mathrm{OH})_{2} \mathrm{D}_{3}$ production [45]. So, Klotho and the vitamin $\mathrm{D}$ endocrine system reciprocally regulate each other (Figure 1). In homozygous $\mathrm{Kl}$-/- mice, extremely high plasma $1,25(\mathrm{OH})_{2} \mathrm{D}_{3}$ levels were noted, as well as up-regulation of 1-a-hydroxylase and downregulation of 24-hydrolase, which provides in vivo genetic, indirect evidence that the high circulating $1,25(\mathrm{OH})_{2} \mathrm{D}_{3}$ levels are the result of overproduction and low degradation of $1,25(\mathrm{OH})_{2} \mathrm{D}_{3}$. The normal genetic responses to vitamin $\mathrm{D}$ supplementation, including downregulation of 1-a-hydroxylase transcripts and up-regulation of 24-hydroxylase and VDR transcripts, were impaired in $\mathrm{Kl}-/$ - mice, suggesting that normal expression of renal klotho is required for normal vitamin $\mathrm{D}$ homeostasis. On the other hand, the dysregulation of the vitamin D system may be associated with high mortality in the Kl-/- mice $[16,17]$.

A primary increase in $1,25(\mathrm{OH})_{2} \mathrm{D}_{3}$ up-regulates klotho expression, which in turn suppresses $1,25(\mathrm{OH})_{2} \mathrm{D}_{3}$ production and likewise an increase in klotho will suppress $1,25(\mathrm{OH})_{2} \mathrm{D}_{3}$ to remove a major stimulator of klotho production Renal la-hydroxylase resulting in a decreased conversion of 25 -hydroxyvitamin $\mathrm{D}$ to $1,25(\mathrm{OH})_{2} \mathrm{D}_{3}$. FGF23 also regulates renal $1,25(\mathrm{OH})_{2} \mathrm{D}_{3}$ levels by inducing expression of the catabolic enzyme 24- hydroxylase. These functions of FGF23 are dependent on the presence of the transmembrane klotho $[16,17,20,23,25]$. 
Citation: Nakhoul F, Nakhoul N, Thaucho N, Farber E, Zhang H, et al. (2015) The Non Mineral Axis Klotho-Vitamin D in Diabetic Nephropathy: Review. J Diabetes Metab 6: 563. doi:10.4172/2155-6156.1000563

Page 4 of 5

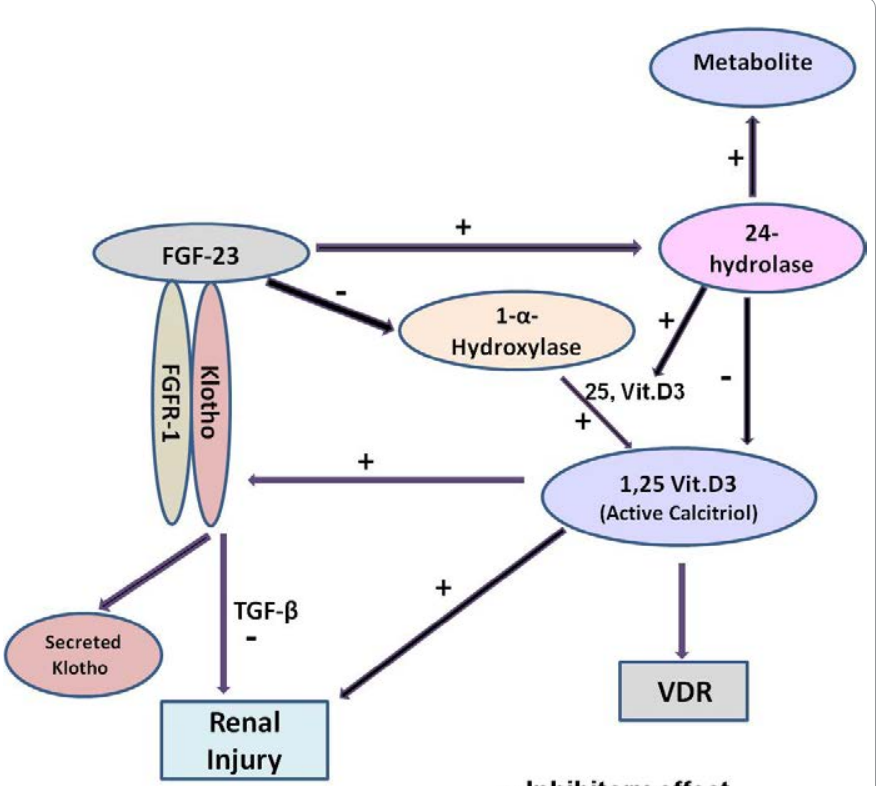

- Inhibitory effect

+ Stimulatory effect

Figure 1: Schematic illustration of the kloto-vitamin D axis cross talk. Normal expression of renal Klotho is required for normal vitamin D homeostasis via down-regulation of 1-a-hydroxylase transcripts and upregulation of 24-hydroxylase. Likewise calcitriol stimulates both Klotho and FGF-23, and both FGF-23 and Klotho inhibits calcitriol. The podocytes express the VDR that is highly inducible by $1,25(\mathrm{OH})_{2} \mathrm{D}_{3}$, and, through VDR, $1,25(\mathrm{OH})_{2} \mathrm{D}_{3}$ transcriptionally stimulates the expression of nephrin, a key slit diaphragm protein synthesized by podocytes. Up regulation or restoration of Klotho by calcitriol may provide a means to slow down the progression of CKD and improve cardiovascular disease especially in the DM patients.

\section{Conclusions}

The potential utility of Klotho in clinical practice is anticipated to be at least two-fold. First, klotho may serve as an early and sensitive biomarker of DN. However its specificity and its prognostic value and differential diagnostic value in human disease remain to be examined. Second, Klotho exogenous supplementation and/or up-regulation of endogenous Klotho production may provide novel therapy for DN patients to retard or block its progression to advanced CKD by arresting or slowing progression as well as preventing and reversing complications. Because we are moving to use klotho as a novel diagnostic, prognostic, and therapeutic strategy for CKD patients, identification of proper indications including CKD stage.

The expression of VDR in kidney tissue was significantly decreased in early stages of $\mathrm{DN}$, and the down-regulation of VDR could be restored to the normal level by the treatment with Paricalcitol, an analog of active vitamin D. More the beneficial effects of vitamin D on renal fibrosis in DN is mediated by VDR via restoration of klotho expression.

\section{References}

1. Zimmet P, Alberti KG, Shaw J (2001) Global and societal implications of the diabetes epidemic. Nature 414: 782-787.

2. Pezzolesi MG, Skupien J, Mychaleckyj JC, Warram JH, Krolewski AS (2010) Insights to the genetics of diabetic nephropathy through a genome-wide association study of the GoKinD collection. Semin Nephrol 30: 126-140.

3. Nakhoul FM, Miller-Lotan R, Awad H, Asleh R, Jad K, et al. (2009) Pharmacogenomic effect of vitamin $\mathrm{E}$ on kidney structure and function in transgenic mice with the haptoglobin 2-2 genotype and diabetes mellitus. Am J Physiol Renal Physiol 296: F830-838.

4. Asleh R, Nakhoul FM, Miller-Lotan R, Awad H, Farbstein D, et al. (2012) Poor lysosomal membrane integrity in proximal tubule cells of haptoglobin 2-2 genotype mice with diabetes mellitus. Free Radic Biol Med 53: 779-786.

5. Tian Y, Lv G, Yang Y, Zhang Y, Yu R, et al. (2014) Effects of vitamin D on renal fibrosis in diabetic nephropathy model rats. Int J Clin Exp Pathol 7: 3028-3037.

6. Zhang Y, Deb DK, Kong J, Ning G, Wang Y, et al. (2009) Long-term therapeutic effect of vitamin $D$ analog doxercalciferol on diabetic nephropathy: strong synergism with AT1 receptor antagonist. Am J Physiol Renal Physiol 297: F791-801.

7. Levy AP (2004) Haptoglobin: a major susceptibility gene for diabetic cardiovascular disease. Isr Med Assoc J 6: 308-310.

8. Park JH, Jang HR, Lee JH, Lee JE, Huh W, et al. (2015) Comparison of intrarenal renin-angiotensin system activity in diabetic versus non-diabetic patients with overt proteinuria. Nephrology (Carlton) 20: 279-285.

9. Li YC (2010) Renoprotective effects of vitamin D analogs. Kidney Int 78: 134 139

10. Tangpricha V, Judd SE, Kamen D, Li YC, Malabanan A (2010) Vitamin d. Int J Endocrinol 2010: 631052.

11. Peng Y, Li LJ (2015) Serum 25-hydroxyvitamin D level and diabetic nephropathy in patients with type 2 diabetes mellitus. Int Urol Nephrol 47: 983-989.

12. Li YC (2014) Discovery of vitamin D hormone as a negative regulator of the renin-angiotensin system. Clin Chem 60: 561-562.

13. Haussler MR, Whitfield GK, Kaneko I, Haussler CA, Hsieh D, et al. (2013) Molecular mechanisms of vitamin D action. Calcif Tissue Int 92: 77-98.

14. Li YC (2013) Vitamin D receptor signaling in renal and cardiovascular protection. Semin Nephrol 33: 433-447.

15. Gysemans C, van Etten E, Overbergh L, Giulietti A, Eelen G, et al. (2008) Unaltered diabetes presentation in NOD mice lacking the vitamin D receptor Diabetes 57: 269-275.

16. Hu MC, Kuro-o M, Moe OW (2013) Renal and extrarenal actions of Klotho Semin Nephrol 33: 118-129.

17. Dërmaku-Sopjani M, Kolgeci S, Abazi S, Sopjani M (2013) Significance of the anti-aging protein Klotho. Mol Membr Biol 30: 369-385.

18. Schmid C, Neidert MC, Tschopp O, Sze L, Bernays RL (2013) Growth hormone and Klotho. J Endocrinol 219: R37-57.

19. Donate-Correa J, Muros-de-Fuentes M, Mora-Fernández C, Navarro-González JF (2013) [Fibroblast growth factor 23/Klotho system in the context of cardiovascular damage]. Med Clin (Barc) 140: 420-423.

20. Hu MC, Kuro-o M, Moe OW (2012) The emerging role of Klotho in clinical nephrology. Nephrol Dial Transplant 27: 2650-2657.

21. Kacso IM, Bondor $\mathrm{Cl}$, Kacso $\mathrm{G}$ (2012) Soluble serum Klotho in diabetic nephropathy: relationship to VEGF-A. Clin Biochem 45: 1415-1420.

22. Mitobe M, Yoshida T, Sugiura H, Shirota S, Tsuchiya K, et al. (2005) Oxidative stress decreases klotho expression in a mouse kidney cell line. Nephron Exp Nephrol 101: e67-74.

23. Xu Y, Sun Z (2015) Molecular basis of Klotho: from gene to function in aging Endocr Rev 36: 174-193.

24. Haruna Y, Kashihara N, Satoh M, Tomita N, Namikoshi T, et al. (2007) Amelioration of progressive renal injury by genetic manipulation of Klotho gene. Proc Natl Acad Sci U S A 104: 2331-2336.

25. Lindberg K, Amin R, Moe OW, Hu MC, Erben RG, et al. (2014) The kidney is the principal organ mediating klotho effects. J Am Soc Nephrol 25: 2169-2175.

26. Hu MC, Shiizaki K, Kuro-o M, Moe OW (2013) Fibroblast growth factor 23 and Klotho: physiology and pathophysiology of an endocrine network of mineral metabolism. Annu Rev Physiol 75: 503-533.

27. Hathaway CK1, Gasim AM1, Grant R1, Chang AS1, Kim HS1, et al. (2015) Low TGF ${ }^{2} 1$ expression prevents and high expression exacerbates diabetic nephropathy in mice. Proc Natl Acad Sci U S A 112: 5815-5820.

28. Zhao Y, Banerjee S, Dey N, LeJeune WS, Sarkar PS, et al. (2011) Klotho 
Citation: Nakhoul F, Nakhoul N, Thaucho N, Farber E, Zhang H, et al. (2015) The Non Mineral Axis Klotho-Vitamin D in Diabetic Nephropathy: Review. J Diabetes Metab 6: 563. doi:10.4172/2155-6156.1000563

Page 5 of 5

depletion contributes to increased inflammation in kidney of the $\mathrm{db} / \mathrm{db}$ mouse model of diabetes via RelA (serine)536 phosphorylation. Diabetes 60: 19071916.

29. Asai O, Nakatani K, Tanaka T, Sakan H, Imura A, et al. (2012) Decreased renal İt-Klotho expression in early diabetic nephropathy in humans and mice and its possible role in urinary calcium excretion. Kidney Int 81: 539-547.

30. Cheng MF, Chen LJ, Cheng JT (2010) Decrease of Klotho in the kidney of streptozotocin-induced diabetic rats. J Biomed Biotechnol 2010: 513853.

31. Lee EY, Kim SS, Lee JS, Kim IJ, Song SH, et al. (2014) Soluble a-klotho as a novel biomarker in the early stage of nephropathy in patients with type 2 diabetes. PLoS One 9: e102984.

32. Kurosu H, Yamamoto M, Clark JD, Pastor JV, Nandi A, et al. (2005) Suppression of aging in mice by the hormone Klotho. Science 309: 1829-1833.

33. Declèves AE, Sharma K (2014) Novel targets of antifibrotic and antiinflammatory treatment in CKD. Nat Rev Nephrol 10: 257-267.

34. Esapa CT, Hannan FM, Babinsky VN, Potter P, Thomas GP, et al. (2015) $\mathrm{N}$-ethyl-N-Nitrosourea (ENU) induced mutations within the klotho gene lead to ectopic calcification and reduced lifespan in mouse models. PLoS One 10: e0122650.

35. Lin Y, Kuro-o M, Sun Z (2013) Genetic deficiency of anti-aging gene klotho exacerbates early nephropathy in STZ-induced diabetes in male mice. Endocrinology 154: 3855-3863.

36. Al-Badr W, Martin KJ (2008) Vitamin D and kidney disease. Clin J Am Soc Nephrol 3: 1555-1560.
37. Li YC, Kong J, Wei M, Chen ZF, Liu SQ, et al. (2002) 1,25-Dihydroxyvitamin $\mathrm{D}(3)$ is a negative endocrine regulator of the renin-angiotensin system. $J$ Clin Invest 110: 229-238.

38. Li YC (2011) Podocytes as target of vitamin D. Curr Diabetes Rev 7: 35-40.

39. Yacoub R, Campbell KN (2015) Inhibition of RAS in diabetic nephropathy. Int J Nephrol Renovasc Dis 8: 29-40.

40. Campbell KN, Raij L, Mundel P (2011) Role of angiotensin II in the development of nephropathy and podocytopathy of diabetes. Curr Diabetes Rev 7: 3-7.

41. Mandarino NR, Júnior Fd, Salgado JV, Lages JS, Filho NS (2015) Is vitamin $\mathrm{d}$ deficiency a new risk factor for cardiovascular disease? Open Cardiovasc Med J 9: 40-49.

42. Dusso AS (2012) Renal vitamin D receptor expression and vitamin D renoprotection. Kidney Int 81: 937-939.

43. Li YC (2003) Vitamin D regulation of the renin-angiotensin system. J Cell Biochem 88: 327-331.

44. Wang Y, Deb DK, Zhang Z, Sun T, Liu W, et al. (2012) Vitamin D receptor signaling in podocytes protects against diabetic nephropathy. J Am Soc Nephrol 23: 1977-1986.

45. Deb DK, Sun T, Wong KE, Zhang Z, Ning G, et al. (2010) Combined vitamin D analog and AT1 receptor antagonist synergistically block the development of kidney disease in a model of type 2 diabetes. Kidney Int 77: 1000-1009. 